\title{
Clustering by phenotype and genome-wide association study in autism
}

\author{
Akira Narita ${ }^{1,2}$, Masato Nagai $\mathbb{1}^{1,2}$, Satoshi Mizuno ${ }^{1,2}$, Soichi Ogishima ${ }^{1,2}$, Gen Tamiya ${ }^{1,2,3}$, Masao Ueki ${ }^{1,2,3}$, \\ Rieko Sakurai 1,2,3, Satoshi Makino ${ }^{1,2,3}$, Taku Obara ${ }^{1,2,4}$, Mami Ishikuro 1,2, Chizuru Yamanaka ${ }^{1,2}$, Hiroko Matsubara 1,2, \\ Yasutaka Kuniyoshi' ${ }^{2}$, Keiko Murakami 1,2, Fumihiko Ueno ${ }^{1,2}$, Aoi Noda ${ }^{1,2,4}$, Tomoko Kobayashi ${ }^{1,2,4}$, Mika Kobayashi', \\ Takuma Usuzaki ${ }^{1}$, Hisashi Ohseto ${ }^{1}$, Atsushi Hozawa ${ }^{1,2}$, Masahiro Kikuya ${ }^{1,2,5}$, Hirohito Metoki ${ }^{1,2,6}$, Shigeo Kure ${ }^{1,2,4}$ and \\ Shinichi Kuriyama (1) ${ }^{1,2,7}$
}

\begin{abstract}
Autism spectrum disorder (ASD) has phenotypically and genetically heterogeneous characteristics. A simulation study demonstrated that attempts to categorize patients with a complex disease into more homogeneous subgroups could have more power to elucidate hidden heritability. We conducted cluster analyses using the k-means algorithm with a cluster number of 15 based on phenotypic variables from the Simons Simplex Collection (SSC). As a preliminary study, we conducted a conventional genome-wide association study (GWAS) with a data set of 597 ASD cases and 370 controls. In the second step, we divided cases based on the clustering results and conducted GWAS in each of the subgroups vs controls (cluster-based GWAS). We also conducted cluster-based GWAS on another SSC data set of 712 probands and 354 controls in the replication stage. In the preliminary study, which was conducted in conventional GWAS design, we observed no significant associations. In the second step of cluster-based GWASs, we identified 65 chromosomal loci, which included 30 intragenic loci located in 21 genes and 35 intergenic loci that satisfied the threshold of $P<5.0 \times 10^{-8}$. Some of these loci were located within or near previously reported candidate genes for ASD: CDH5, CNTN5, CNTNAP5, DNAH17, DPP10, DSCAM, FOXK1, GABBR2, GRIN2A5, ITPR1, NTM, SDK1, SNCA, and SRRM4. Of these 65 significant chromosomal loci, rs 11064685 located within the SRRM4 gene had a significantly different distribution in the cases vs controls in the replication cohort. These findings suggest that clustering may successfully identify subgroups with relatively homogeneous disease etiologies. Further cluster validation and replication studies are warranted in larger cohorts.
\end{abstract}

\section{Introduction}

Autism spectrum disorder (ASD) has heterogeneous characteristics in terms of both phenotypic features and genetics. ASD is mainly characterized by difficulties in communication and repetitive behaviors, but ASD also shows many other symptoms ${ }^{1}$. Regarding genetics, previous studies have not consistently identified genetic variants that are associated with an increased risk of

\footnotetext{
Correspondence: Shinichi Kuriyama (kuriyama@med.tohoku.ac.jp)

${ }^{1}$ Tohoku Medical Megabank Organization, Tohoku University, Sendai, Japan

${ }^{2}$ Graduate School of Medicine, Tohoku University, Sendai, Japan

Full list of author information is available at the end of the article

These authors contributed equally: Akira Narita, Masato Nagai, Satoshi Mizuno
}

$\mathrm{ASD}^{2}$, although several lines of evidence suggest that genetic factors strongly contribute to the increased risk of ASD. Monozygotic twins have higher concordance rates of ASD (92\%) than dizygotic twins $(10 \%)^{3}$. The recurrence risk ratio is 22 for ASD among siblings ${ }^{4}$. The Human Gene module of the Simons Foundation Autism Research Initiative (SFARI) gene provides a comprehensive reference for suggested human ASD-related genes in an up-to-date manner ${ }^{5}$ and currently demonstrates $\sim 1000$ genes that may have links to ASD, potentially indicating the heterogeneity of ASD. In addition to phenotype and genotype heterogeneities, ASD shows heterogeneous responses to interventions. Several kinds of pharmacological 
treatments are suggested, but the effects of these treatments are controversial ${ }^{6}$.

If the heterogeneous phenotypes and responses to treatment in some way correspond to differences in genotype, grouping persons with ASD according to phenotype and responses to treatment variables may increase the chances of identifying genetic susceptibility factors. Traylor and colleagues ${ }^{7}$ demonstrated that attempts to categorize patients with a complex disease into more homogeneous subgroups could have more power to elucidate the hidden heritability in a simulation study. Several studies on Alzheimer's disease, neuroticism, or asthma indicated that items or symptoms were to some degree more useful for identifying high-impact genetic factors than broadly defined diagnoses ${ }^{8-10}$, although a study of ASD demonstrated modest effects of two-way stratification by individual symptoms ${ }^{11}$. In addition, medical researchers have begun to use machine learning methods, which is an artificial intelligence technique that can reveal masked patterns of data sets. In view of the abovementioned circumstances, clustering algorithms of machine learning and subsequent genome-wide association studies (GWASs) could be hypothesized to reveal novel and more genetically homogeneous clusters, but a combinatorial approach of cluster analysis and GWASs, to the best of our knowledge, has not been applied to any diseases including ASD.

We therefore explored whether grouping persons with ASD using a clustering algorithm with phenotype and responses to treatment variables can be used to discriminate more genetically homogeneous persons with ASD. In the present study, we conducted cluster-based GWASs (named cluster-based GWASs) using real data based on the concept of a previous simulation study ${ }^{7}$ adopting a machine learning $\mathrm{k}$-means ${ }^{12}$ algorithm for cluster analysis.

\section{Subjects and methods}

We conducted the present study in accordance with the guidelines of the Declaration of Helsinki ${ }^{13}$ and all other applicable guidelines. The protocol was reviewed and approved by the institutional review board of Tohoku University Graduate School of Medicine, and written informed consent was obtained from all participants over the age of 18 by the SFARI ${ }^{14}$. For participants under the age of 18, informed consent was obtained from a parent and/or legal guardian. In addition, for participants 10-17 years of age, informed assent was obtained from the individuals.

\section{data sets}

We used phenotypic variables, history of treatment, and genotypic data from the Simons Simplex Collection $(\mathrm{SSC})^{14}$. The SSC establishes a repository of phenotypic data and genetic data/samples from mainly simplex families.
The SSC data were publicly released in October 2007 and are directly available from the SFARI. From the SSC data set, we used data from 614 affected white male probands who had no missing information regarding Autism Diagnostic Interview-Revised (ADI-R) scores $^{15}$ and vitamin treatment ${ }^{16,17}$ and 391 unaffected brothers for whom genotype data, generated by the Illumina Human Omni2.5 (Omni2.5) array, were available for subsequent clustering and genetic analyses. We excluded participants whose ancestries were estimated to be different from the other participants using principal component analyses (PCAs) performed by EIGENSOFT version $7.2 .1^{18}$ for the genotype data. Based on the PCAs, we excluded data beyond four standard deviations of principal components 1 or 2 (Supplementary Fig. 1). Therefore, we used data from 597 probands and 370 unaffected brothers.

In the replication study, we used another SSC data set genotyped using the Illumina $1 \mathrm{Mv} 3(1 \mathrm{Mv} 3)$ array. In the data set, data from 735 affected male probands with no missing information regarding ADI-R scores or vitamin treatment and 387 unaffected brothers were available. After conducting PCA, we excluded data beyond four standard deviations of principal components 1 or 2 as outliers. In this way, we used data from 712 probands and 354 unaffected brothers in the replication study.

\section{Clustering}

We conducted cluster analyses using phenotypic variables of ADI-R scores and history of vitamin treatment ${ }^{16,17}$. We chose these variables because the ADI-R is one of the most reliable estimates of ASD and has the ability to evaluate substructure domains of $\mathrm{ASD}^{15}$. Among the ADI-R scores, "the total score for the Verbal Communication Domain of the ADI-R minus the total score for the Nonverbal Communication Domain of the ADIR", "the total score for the Nonverbal Communication Domain of the ADI-R", "the total score for the Restricted, Repetitive, and Stereotyped Patterns of Behavior Domain of the ADI-R", and "the total score for the Reciprocal Social Interaction Domain of the ADI-R" were included in the preprocessed data set.

Among the treatments, we selected the variable of history of vitamin treatment because we recently found that a cluster of persons with ASD is associated with potential responsiveness to vitamin $\mathrm{B} 6$ treatment $^{16,17}$. The history of treatment is not always compatible with responsiveness, but we considered that continuous treatment indicates responsiveness to some degree. The SSC data set includes history of treatment but not variables of responsiveness.

We applied the machine learning k-means ${ }^{12}$ algorithm to conduct a cluster analysis to divide the data set obtained from ASD persons into subgroups using 
phenotypic variables and history of treatment. The $\mathrm{k}$-means algorithm requires a cluster number $(\mathrm{k})$ determined by researchers. We set a priori $k$ of $5,10,15$, and 20 under the hypothesis that ASD consists of hundreds of subgroups $^{5,14}$ and considering statistical power by sample size calculations ${ }^{19}$. We performed the analyses using the scikit-learn toolkit in Python 2.7 (Supplementary Information 1).

Clustering is an exploratory data analysis technique, and the validity of the clustering results may be judged by external knowledge, such as the purpose of the segmentation $^{20}$. Several methods have proposed to prespecify a cluster number of $\mathrm{k}$, such as visual examination of the data, and likelihood and error-based approaches; however, these methods do not necessarily provide results that are consistent with each other ${ }^{21}$. Although there are measures for evaluating the quality of the clusters ${ }^{22}$, the number of clusters should still be determined according to the research purposes. We regarded the inflation factor $(\lambda)$ of quantile-quantile $(\mathrm{Q}-\mathrm{Q})$ plots of the logarithm of the $P$ value to base $10\left(-\log _{10} P\right)$ as one of the indicators of successful clustering in the present study. We calculated $\lambda$ for each cluster number.

When conducting clustering, we combined the two data sets of male probands, one genotyped using the Omni2.5 array and the other genotyped using the $1 \mathrm{Mv} 3$ array. After clustering, we redivided the new data set according to the SNP arrays used. In the discovery stage, we used the Omni2.5 data set and the $1 \mathrm{Mv} 3$ data set in the replication stage.

\section{Genotype data and quality control}

We used the SSC data set, in which probands and unaffected brothers had already been genotyped in other previous studies ${ }^{14,23}$. In the discovery stage, we used the data set genotyped by the Omni2.5 array, which has 2,383,385 probes. We excluded SNPs with a minor allele frequency $<0.01$, call rate $<0.95$, and Hardy-Weinberg equilibrium test $P<0.000001$.

In the replication study, where we used the data set genotyped using the $1 \mathrm{Mv} 3$ array, we applied the same cutoff values for quality control as those used in the discovery stage. The $1 \mathrm{Mv} 3$ array includes $1,147,689$ SNPs. The Omni2.5 array and the $1 \mathrm{Mv} 3$ array shared 675,923 SNPs.

\section{Statistical analysis}

As a preliminary study, we conducted a conventional GWAS in the whole Omni2.5 data set, with a total of 597 male probands and 370 unaffected brothers. Here, we used the brothers of the cases as controls, in contrast to many previous studies in which genetically unrelated controls were used. We thus adopted the sib transmission disequilibrium test (sib-TDT) $)^{24}$, a family-based association test, to take into account familial relationships among the participants. In the second step, in the discovery stage, we conducted cluster-based GWAS in each subgroup of the cases, which had been divided using the k-means ${ }^{12}$ algorithm, and the controls. As mentioned above, the controls were the brothers of the cases, and we then excluded the unaffected brothers of the cases belonging to the subgroup being analyzed. Details of the study design are shown in Supplementary Fig. 2. We applied the Cochran-Armitage trend test ${ }^{25}$, which examines the risk of disease in those who do not have the allele of interest, those who have a single copy, and those who are homozygous.

We further tested the significantly associated loci found in the discovery studies in the replication stage. The level of significance for association was set as $P<0.05$ in the replication studies.

Association analyses were performed with the PLINK software package ${ }^{26}$. The detected SNPs were subsequently annotated using ANNOVAR ${ }^{27}$. Manhattan plots and $\mathrm{Q}-\mathrm{Q}$ plots were generated using the 'qqman' package in $\mathrm{R}$ version 3.0.2.

\section{Results \\ Cluster-based GWAS}

As a preliminary study, we conducted a conventional GWAS with the Omni2.5 data set using the sib-TDT. We observed no significant associations (Fig. 1). Although we adopted the sib-TDT here because we used the brothers of the cases as controls, we also used the Cochran-Armitage trend test and found that the $-\log _{10} P$ values were distributed downward compared with the expected values, as shown in Supplementary Fig. 3.

We also applied the sib-TDT to cluster 1 , which was obtained by dividing all the cases using $\mathrm{k}$-means with $k$ of 15 , and all the controls and found that the observed $-\log P$ values were lower than expected, as shown in Supplementary Fig. 3. As the sib-TDT may efficiently work in a population consisting of a substantial number of sibs, a limited number of brothers of the probands among all the controls probably contributed to a substantial loss of power. Thus, we excluded the brothers of the probands in each subset from the controls so that each subset of probands has no genetic relations with the rest of the controls and conducted the Cochran-Armitage trend test, as in many other studies. In the present study, therefore, we applied the sib-TDT to the GWAS of the whole data set, whereas in the cluster-based GWAS, we excluded in turn the unaffected brothers of the cases belonging to the subgroup being analyzed and used the Cochran-Armitage trend test to account for the relationships between participants.

The average inflation factor $\lambda$ for the cluster-based GWAS with $\mathrm{k}$ of $5,10,15$, and 20 were 1.021, 1.024, 1.038, and 1.053, respectively. Several lines of evidence suggest that regarding an appropriate threshold of $\lambda$, empirically, a 


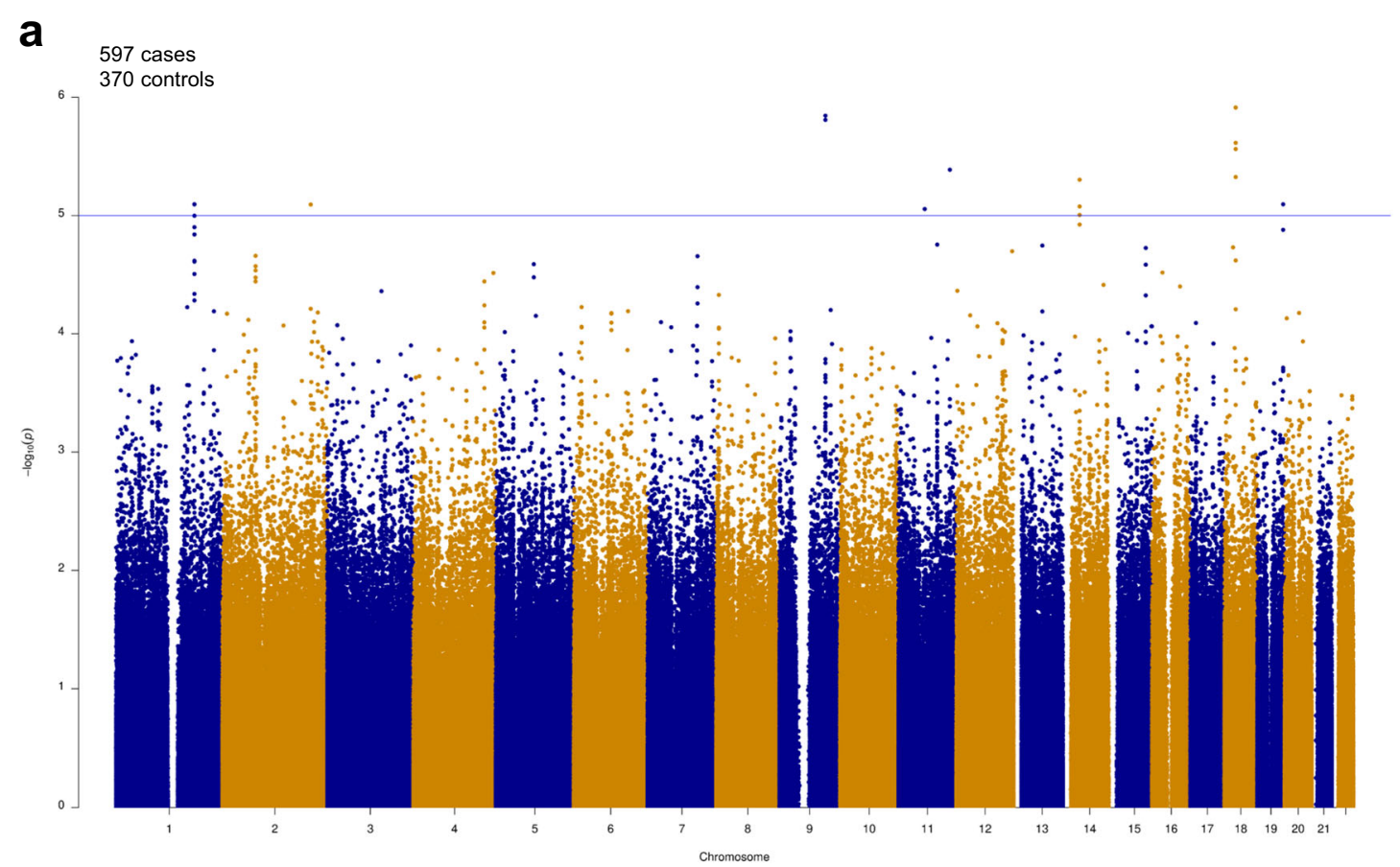

b

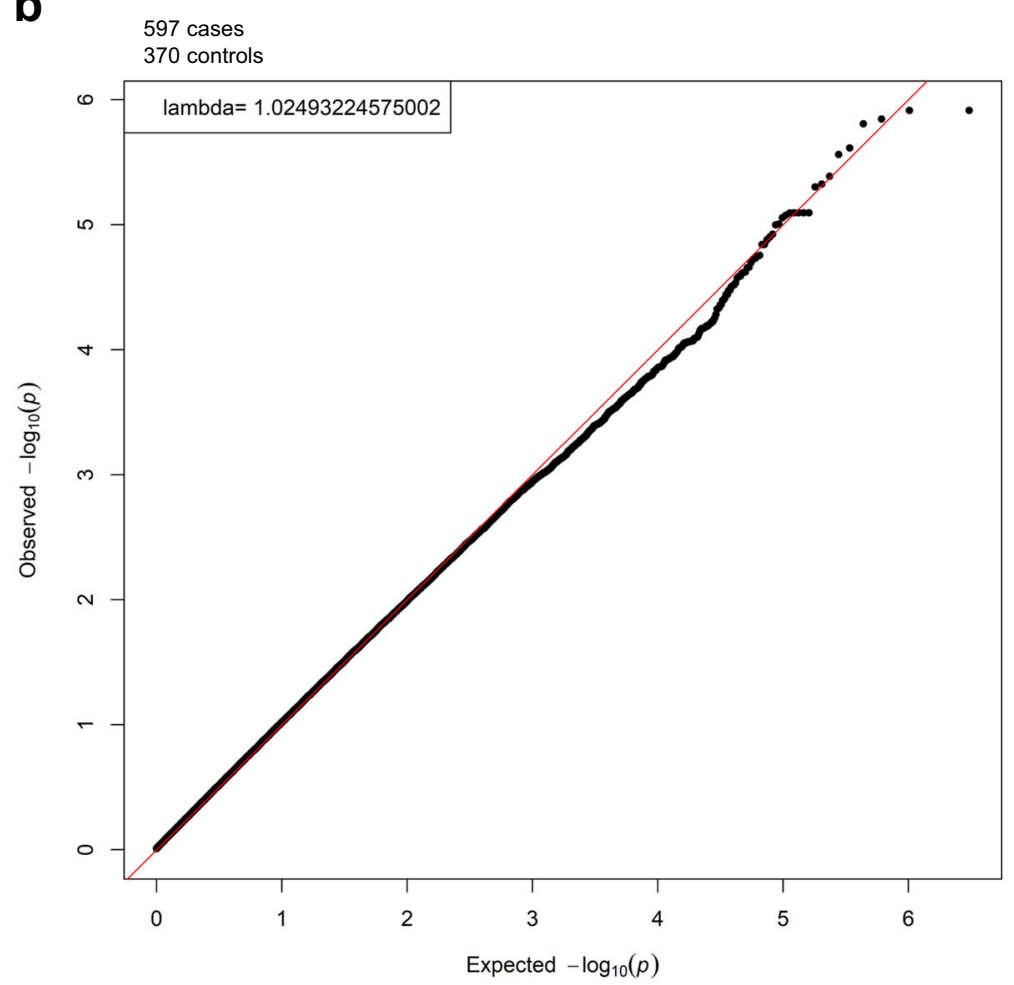

Fig. 1 Manhattan plot and corresponding quantile-quantile plot in GWAS for all male probands vs their unaffected brothers. Manhattan plot $\mathbf{a}$ and corresponding quantile-quantile plot $\mathbf{b}$ in GWAS for all male probands vs their unaffected brothers. We conducted a GWAS in the Simons Simplex Collection data set of 597 male probands and 370 unaffected brothers genotyped by the Illumina Human Omni2.5 array using the sib transmission/disequilibrium test (sib-TDT). We observed no significant associations in this GWAS with the genome-wide threshold of $P=5.0 \times 10^{-8}$. The blue horizontal line indicates the genome-wide suggestive threshold of $p=1.0 \times 10^{-5}$. 


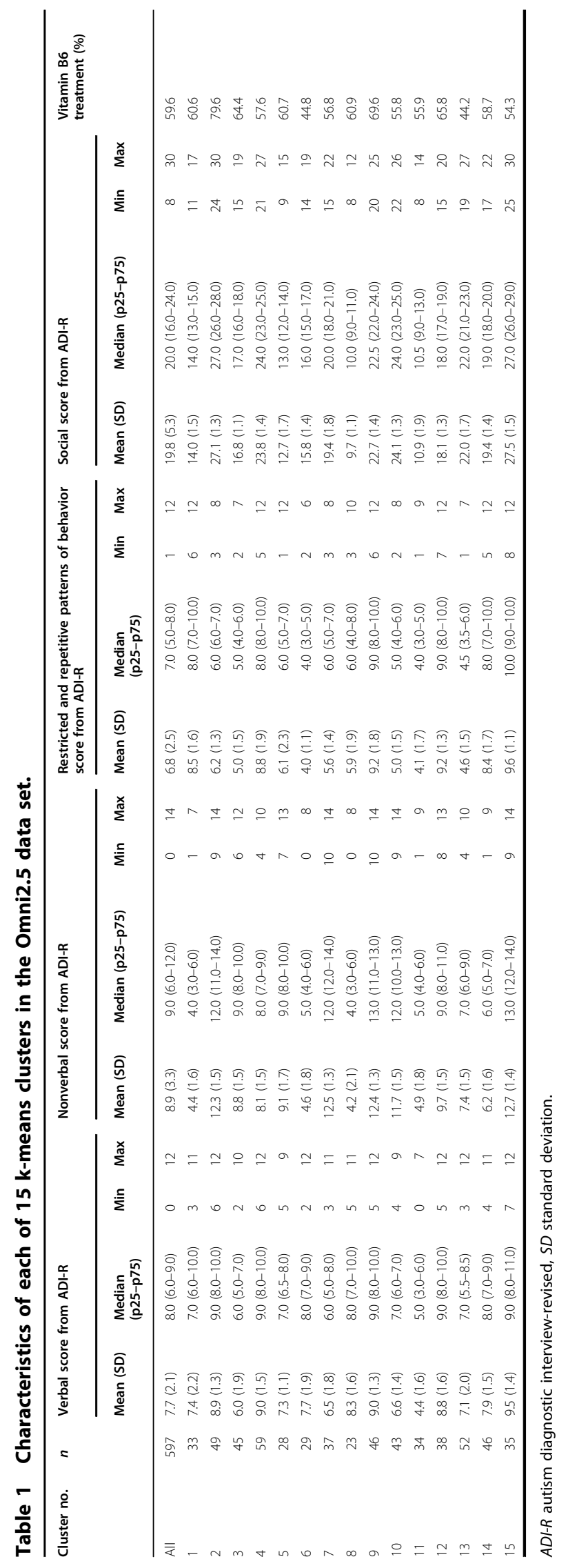

value $<1.050$ is deemed safe for avoiding false positives ${ }^{28}$. Under the hypothesis that ASD consists of hundreds of subgroups ${ }^{14}$, we compared $\lambda$ values giving larger numbers of clusters as priority. We therefore considered the cluster-based GWAS using k-means cluster analysis with $k$ of 15 to be the most appropriate approach to the present data set. The characteristics of each cluster are presented in Table 1.

\section{Gene interpretation}

We observed 65 chromosomal loci that satisfied the threshold of $P<5.0 \times 10^{-8}$ (Fig. 2); 30 out of the 65 loci were located within 21 genes, and the remaining 35 loci were intergenic (Table 2). Among them, eight loci were located within or near the genes associated with the Human Gene module of the SFARI Gene scoring system ${ }^{5}$; GABBR2 (score 4, Rare Single Gene Mutation, Syndromic, Functional) in Cluster 1; CNTNAP5 (score 4, Rare Single Gene Mutation, Genetic Association) in Cluster 3; ITPR1 (score 4, Rare Single Gene Mutation) in Cluster 5; DNAH17 (score 4, Rare Single Gene Mutation) in Cluster 7; $S D K 1$ (score none, Rare Single Gene Mutation, Genetic Association) in Cluster 13; SRRM4 (score 5, Rare Single Gene Mutation, Functional) in Cluster 13; CNTN5 (score 3, Rare Single Gene Mutation, Genetic Association) in Cluster 14; and DPP10 (score 3, Rare Single Gene Mutation) in Cluster 15.

The SFARI Gene scoring system ranges from "Category 1 ", which indicates "high confidence", through "Category 6 ", which denotes "evidence does not support a role". Genes of a syndromic disorder (e.g., fragile X syndrome) related to ASD are categorized in a different category. Rare single-gene variants, disruptions/mutations, and submicroscopic deletions/duplications related to ASD are categorized as "Rare Single Gene Mutation".

In addition to genes in the Human Gene module of the SFARI Gene, several important genes associated with ASD or other related disorders ${ }^{29}$ from previous reports were included in our findings as follows: $C D H 5$ in Cluster 14, DSCAM in Cluster 8, FOXK1 in Cluster 13, GRIN2A in Cluster 5, NTM in Cluster 8, and SNCA in Cluster 11 previously reported with $\mathrm{ASD}^{30-35}$; $\mathrm{PLCH} 2$ in Cluster 11 previously reported with mental retardation ${ }^{36}$; ARHGAP18 in Cluster 18, CDC42BPA in Cluster 3, CXCL12 in Cluster 8 , and HS3ST2 in Cluster 5 previously reported with schizophrenia ${ }^{37-40}$; KCTD12 in Cluster 9 and PSAT1 in Cluster 8 previously reported with depressive disorder ${ }^{41,42}$; and ADAMTS1 in Cluster 10, DOCK2 in Cluster 10, HS3ST2 in Cluster 5, NAMPT in Cluster 5, and NAV in Cluster 5 previously reported with Alzheimer's disease ${ }^{43-47}$.

\section{Replication study}

We conducted replication studies with another independent data set that included a total of 712 male 

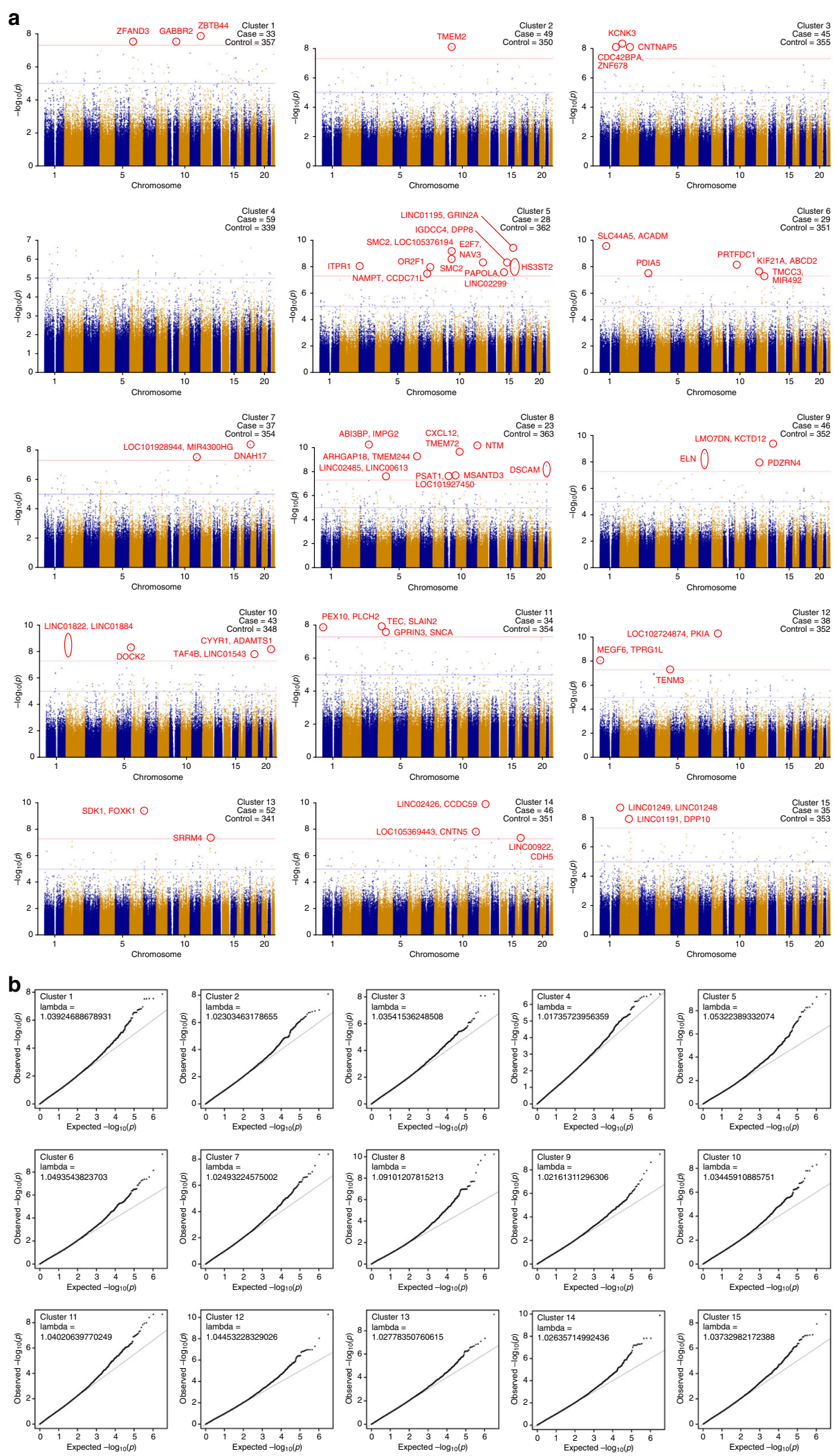

Fig. 2 Manhattan plots and corresponding quantile-quantile plots in cluster-based GWASs. Manhattan plots a and corresponding quantilequantile plots $\mathbf{b}$ in cluster-based GWASs with a cluster number of 15 . We performed cluster analysis using k-means with a cluster number of 15 and conducted cluster-based GWAS. Among 15 clusters, significant associations were observed in 14 clusters. In total, we observed 65 chromosomal loci, labeled in the figure, that satisfied the threshold of $P=5.0 \times 10^{-8}$. The red horizontal lines indicate the threshold for genome-wide significance $(P=5.0 \times$ $\left.10^{-8}\right)$ and the blue horizontal lines indicate the genome-wide suggestive threshold $\left(P=1.0 \times 10^{-5}\right)$. The names of the suggested genes where the excerpted and circled SNPs are located are typed in Manhattan plots. 


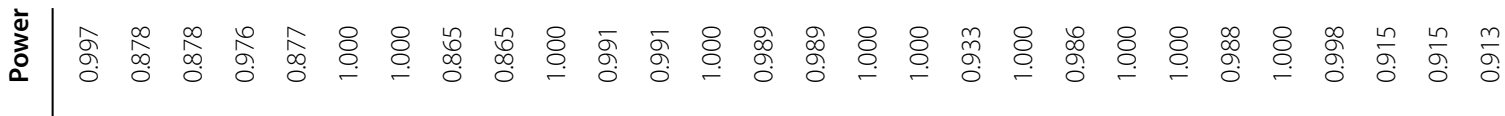

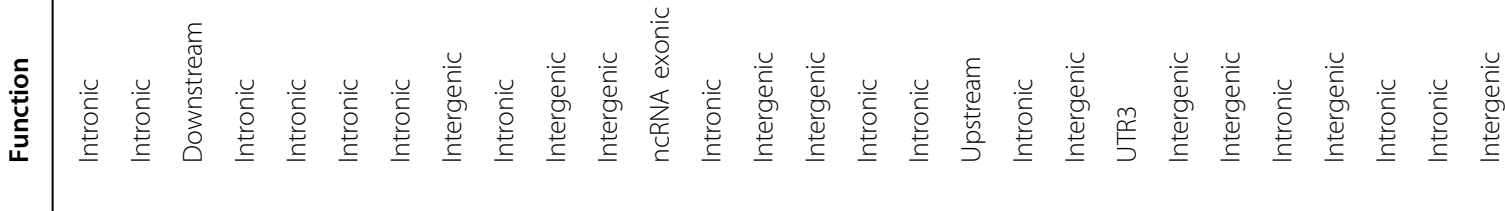

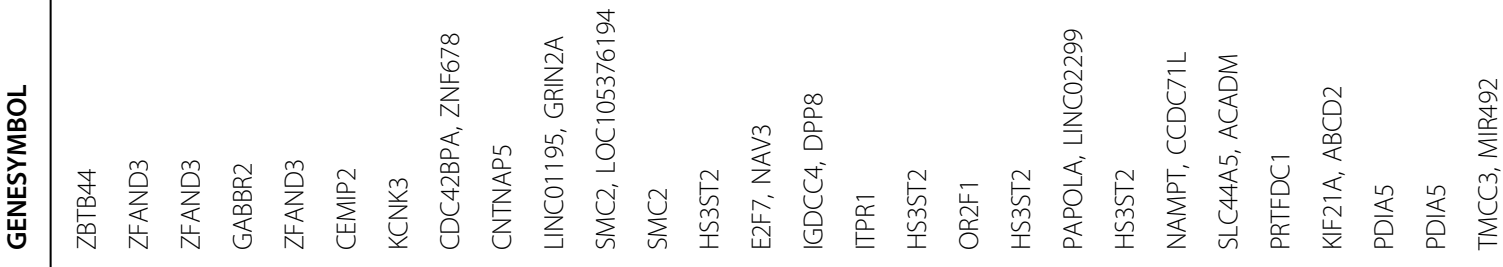

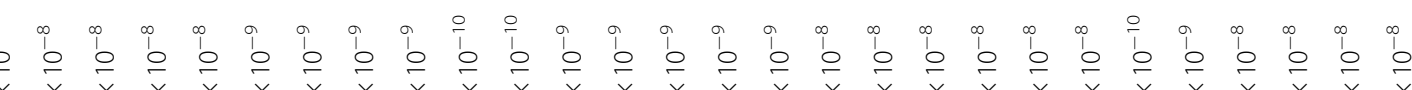

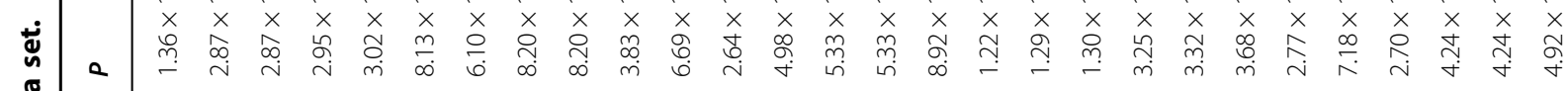

\section{要}

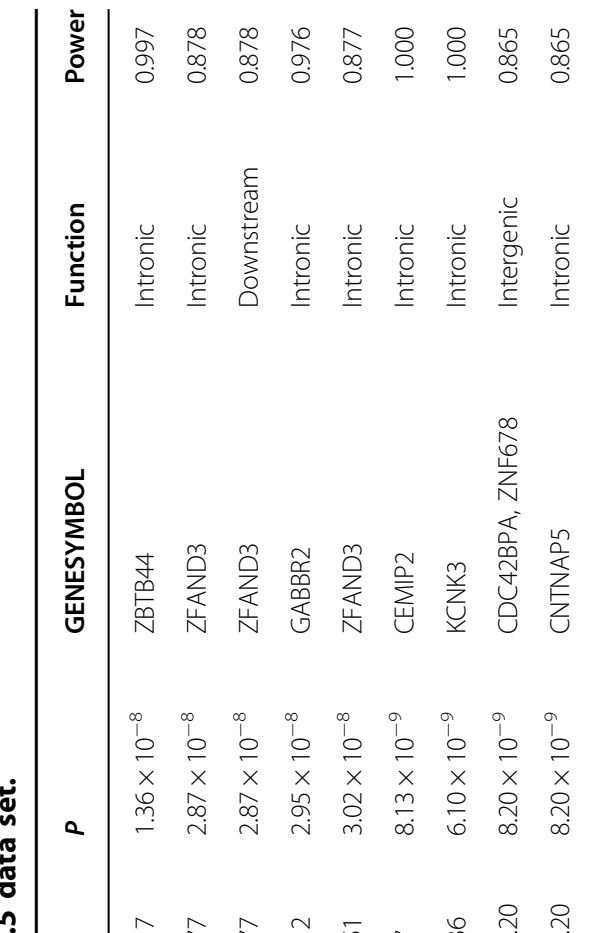

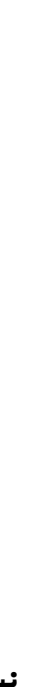

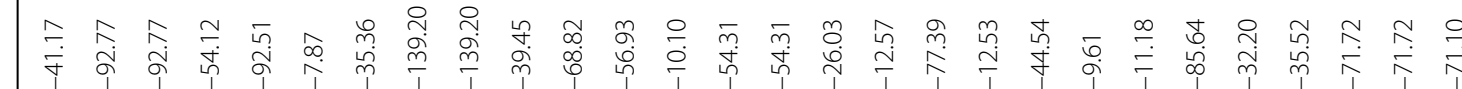

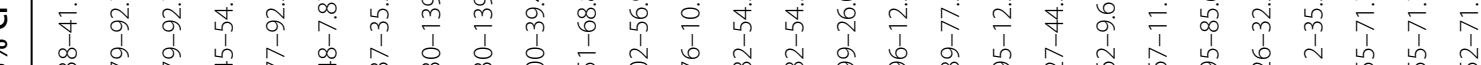

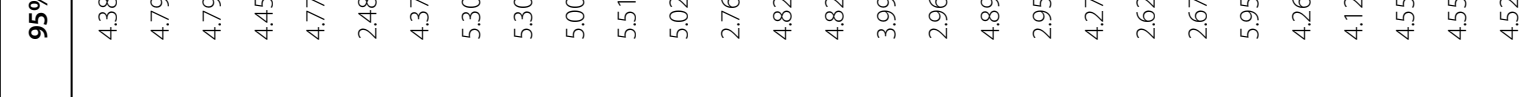

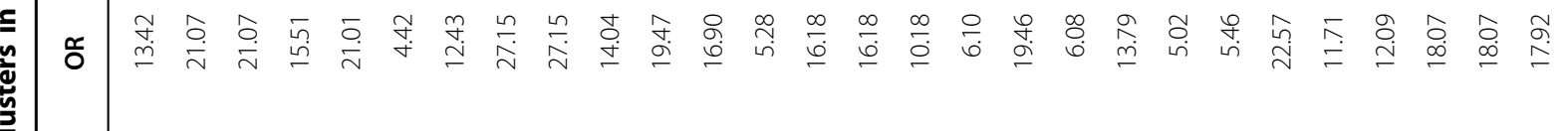

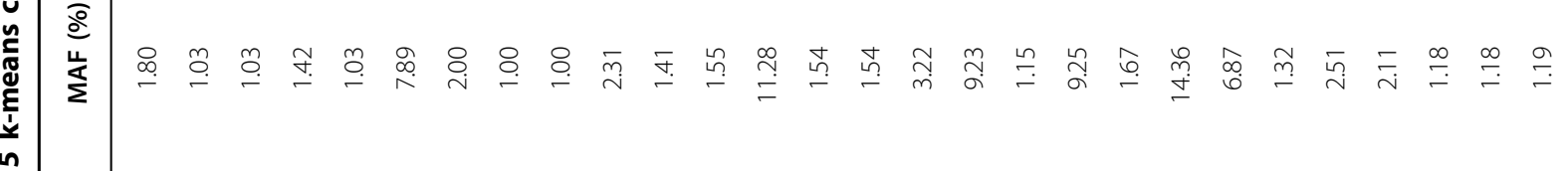

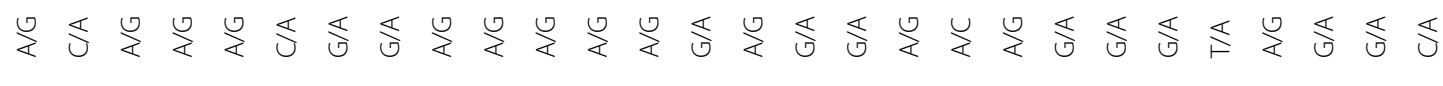

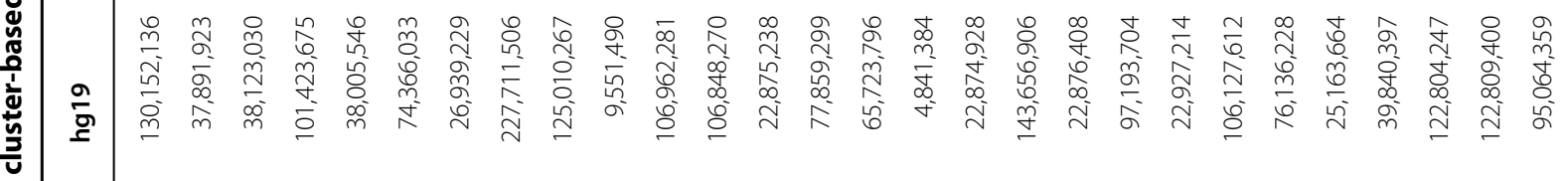

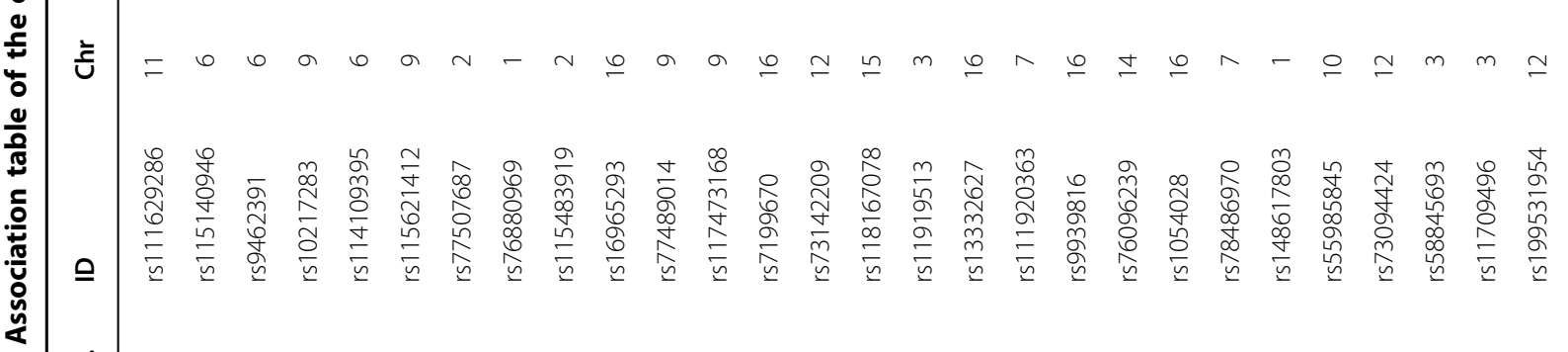




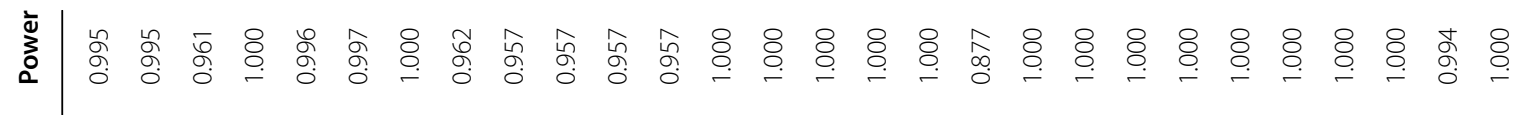

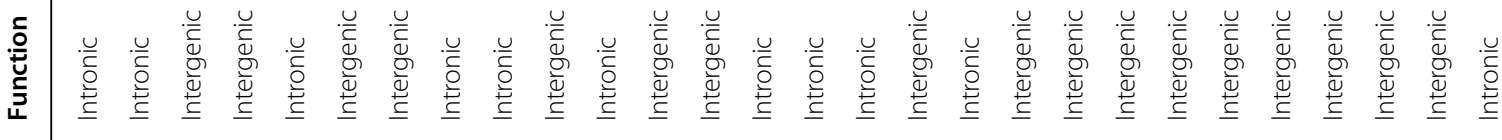

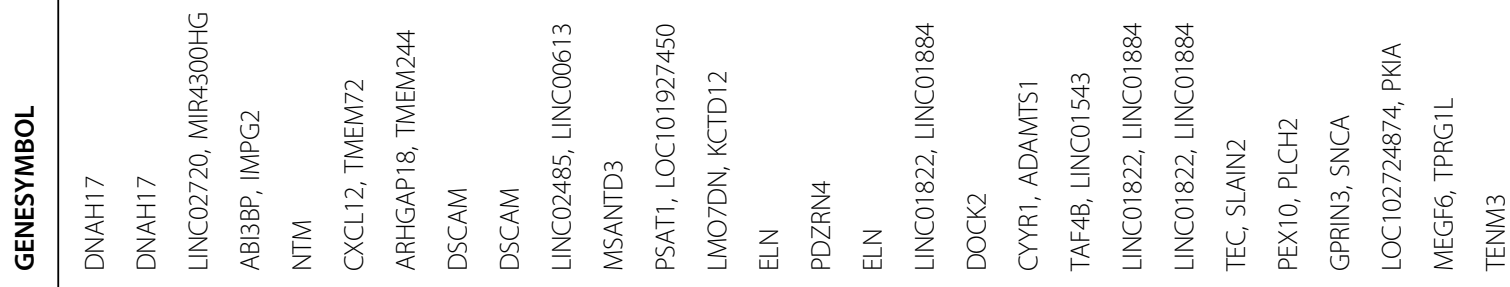

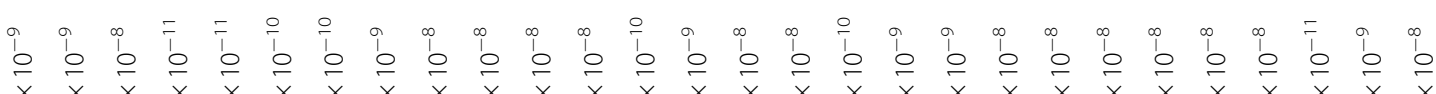

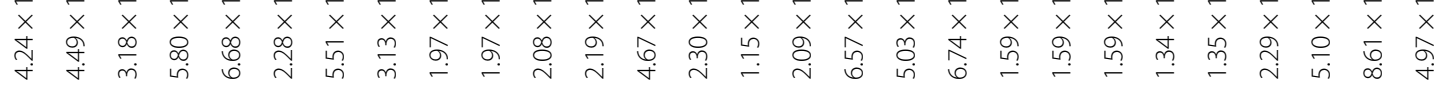

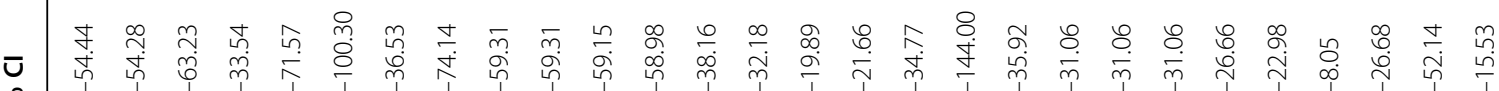

管

\&

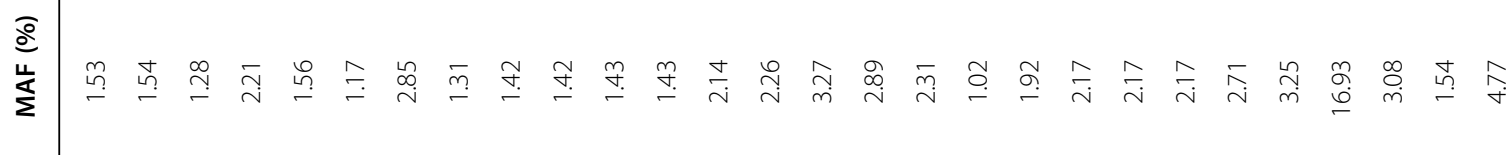

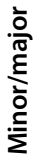

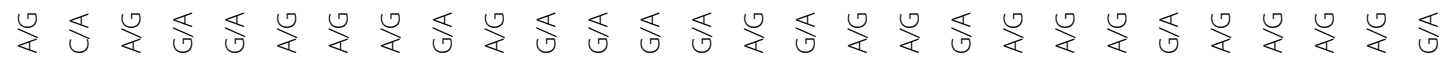

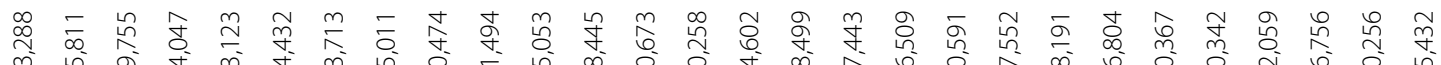

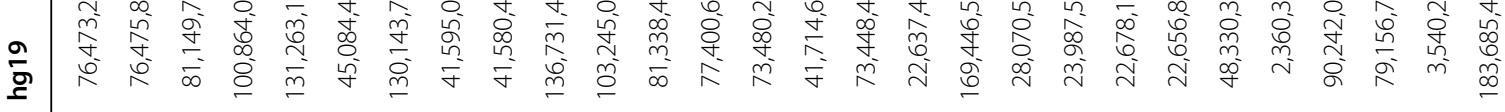

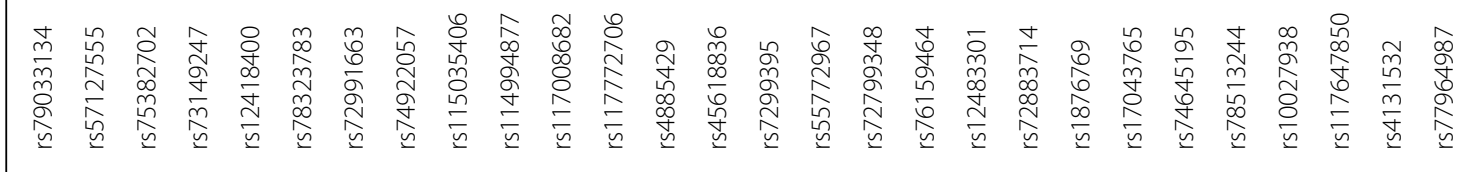




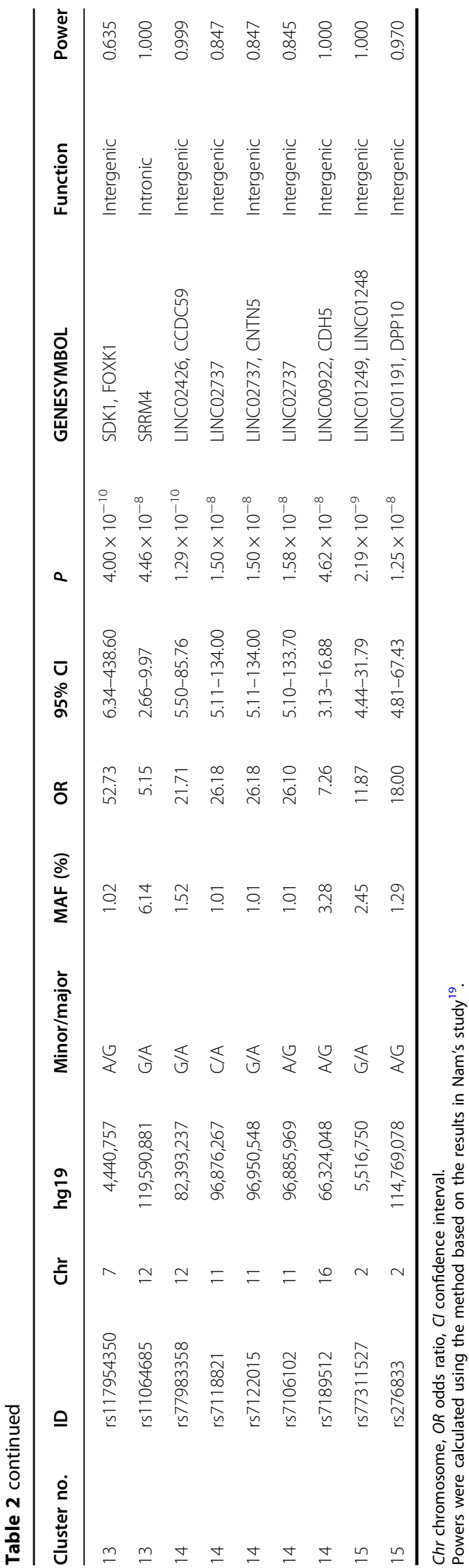

probands and 354 unaffected brothers and had been genotyped using the $1 \mathrm{Mv} 3$ array. As mentioned before, we had previously carried out cluster analyses in the combined data set genotyped with either Omni2.5 or $1 \mathrm{Mv} 3$ and then redivided it according to the SNP arrays used. The characteristics of each of the 15 clusters in the $1 \mathrm{Mv} 3$ data set are presented in Supplementary Table 1.

Among the 65 genome-wide significant chromosomal loci found in the discovery study, seven chromosomal loci were included in the $1 \mathrm{Mv} 3$ array. Of these loci, rs11064685, within SRRM4 in Cluster 13, had a significantly different distribution $(p=0.03)$ in cases vs controls in the replication cohort (Table 3).

\section{Discussion}

One of the most important findings of our study was that reasonably decreasing the sample size could increase the statistical power. A plausible explanation is that our clustering may have successfully identified subgroups that are etiologically more homogeneous. At least two reasons could reduce the possibility of false positives of the present results of statistically significant SNPs in clusterbased GWAS. First, the present study validated the usefulness and feasibility of the concept of a previous simulation study ${ }^{7}$, which indicated that homogeneous case subgroups increase power in genetic association studies by Traylor and colleagues, using measurement data in the real world. Second, a substantial number of statistically significant SNPs in cluster-based GWAS observed in the present study were located within or near previously reported candidate genes for $\mathrm{ASD}^{5,30-35}$.

We observed many statistically significant SNPs in cluster-based GWAS: CDH5, CNTN5, CNTNAP5, DNAH17, DPP10, DSCAM, FOXK1, GABBR2, GRIN2A5, ITPR1, NTM, SDK1, SNCA, and SRRM4. In particular, loci within the SRRM4 gene had significantly different distributions in the cases vs controls in the replication cohort. Previous studies indicate that SRRM4 is strongly associated with ASD, indicating that our results may be valid to some degree. The gene regulates neural microexons. In the brains of individuals with ASD, these microexons are frequently dysregulated ${ }^{48}$. In addition, nSR100/SRRM4 haploinsufficiency in mice induced autistic features such as sensory hypersensitivity and altered social behavior and impaired synaptic transmission and excitability ${ }^{49}$.

In addition to SRRM4, we observed several genes located within or near previously reported candidate genes for ASD. The relatively high correspondence between our results in part and the SFARI Gene scoring system ${ }^{5}$ indicates that the statistically significant loci we found may be associated with ASD subgroups (Fig. 2). We also observed several important genes associated with ASD and other related disorders ${ }^{29}$ from previous reports. 
Table 3 Results of replication studies in the $1 \mathrm{Mv} 3$ data set for statistically significant chromosomal loci in the discovery studies.

\begin{tabular}{lllllllllll}
\hline Cluster no. & ID & Chr & hg19 & Minor/major & MAF (\%) & OR & $\mathbf{9 5 \%}$ Cl & $\boldsymbol{P}$ & GENESYMBOL & Function \\
\hline 5 & rs13332627 & 16 & $22,874,928$ & G/A & 10.0 & & 0.50 & & $0.18-1.45$ & 0.195 \\
5 & rs7199670 & 16 & $22,875,238$ & A/G & 12.2 & 0.51 & $0.20-1.33$ & 0.1629 & HS3ST2 & Intronic \\
5 & rs1054028 & 16 & $22,927,214$ & G/A & 15.0 & 0.51 & $0.22-1.21$ & 0.121 & HS3ST2 & UTR3 \\
10 & rs1876769 & 2 & $22,678,191$ & A/G & 1.4 & NA & - & 0.1822 & LINC01822, LINC01884 & Intergenic \\
13 & rs11064685 & 12 & $119,590,881$ & G/A & 8.2 & 1.89 & $1.06-3.37$ & 0.02858 & SRRM4 & Intronic \\
14 & rs7189512 & 16 & $66,324,048$ & A/G & 3.5 & 2.16 & $0.83-5.67$ & 0.1085 & LINC00922, CDH5 & Intergenic \\
15 & rs276833 & 2 & $114,769,078$ & A/G & 1.3 & 0.71 & $0.09-5.75$ & 0.75 & LINC01191, DPP10 & Intergenic \\
\hline
\end{tabular}

Chr chromosome, $\mathrm{OR}$ odds ratio, $\mathrm{Cl}$ confidence interval.

These findings suggest that the statistically significant SNPs might explain autistic symptoms because these diseases are suggested to have shared etiology, even in part, with $\mathrm{ASD}^{29}$. Associations at the remaining significant loci that were not in the SFARI module or described above have not been previously reported, and to the best of our knowledge, some of them might be novel findings. These results might suggest that novel genetic loci of ASD could be found by identifying better defined subgroups, although further confirmation is needed in future cohorts with larger sample sizes.

Previous studies regarding Alzheimer's disease, neuroticism, or asthma found that items or symptoms showed, to some degree, increased ORs between the case loci and control loci compared with those from previous studies using broadly defined disease diagnoses ${ }^{8-10}$. These findings may indicate that GWAS based on a symptom or an item could identify genetically more homogeneous subgroups and let us hypothesize that a relatively reasonable combination of symptoms or items could identify more genetically homogeneous subgroups. In contrast, Chaste and colleagues showed that stratifying children with ASD based on the phenotype only modestly increased power in GWAS $^{11}$. The discrepancy between their findings and ours might be explained by usage of phenotype variables. Chaste and colleagues used one item or symptom alone with limited number of subgroups, whereas we used combinations of them with a machine learning method with a potentially sufficient number of clusters. DeMichele-Sweet and colleagues reported that subgrouping only by having psychosis could lead to the identification of limited loci that had small effects ${ }^{50}$, but Mukherjee and colleagues found a substantial number of suggestive loci that had extreme ORs after categorizing persons with Alzheimer's disease based on relative performance across cognitive domains by modern psychometric approaches ${ }^{8}$.

Validation of clusters is essential. In the present study, we selected the k-means algorithm, focused on ADI-R items and treatment as variables, and determined cluster numbers based on the $\lambda$ of the $\mathrm{Q}-\mathrm{Q}$ plots. Although we believe this approach is one of the relevant ways, selection of variables, selection of algorithms and selection of cluster numbers still remain to be considered in future mathematical and biological cluster validation studies because controversies surrounding evaluation of the quality of the clusters are important issues and are still ongoing and because validated clusters may lead to elucidate the genetic architectures of $\mathrm{ASD}^{7}$.

The present study has a limitation to be noted. Substantial differences in the two genotyping platforms may have affected the results of the replication study. The Omni2.5 array includes 2,383,385 autosomal SNPs, whereas the $1 \mathrm{Mv} 3$ array includes $1,147,689$ SNPs, with 675,923 shared SNPs between the two. Of the 65 statistically significant chromosomal loci in the discovery data, only seven chromosomal loci were shared between the two arrays.

Our study demonstrated that if the data set consists of multiple heterogeneous subgroups, even a subgroup that includes a much smaller number of homogeneous individuals could detect high-impact genetic factors. Hypothetical examples of the concept of cluster-based GWAS are shown in Supplementary Fig. 4. As shown in Table 2, only 30 etiologically homogeneous probands and 300 controls can have a statistical power of $\sim 1.00$, calculated using the method based on the results in Nam's study ${ }^{19}$. Although the integral model, which assumes many genetic variants have a small effect, may contribute to the formation of some subgroups of ASD, our results indicate that clustering by specific phenotypic variables may provide a candidate example for identifying etiologically similar cases of ASD.

Our data indicate the relevance of cluster-based GWAS as a means to identify more homogeneous subgroups of ASD than broadly defined subgroups. Future investigation of cluster validation and replication with a larger sample 
size is therefore warranted. Such studies will provide clues to elucidate the genetic structures and etiologies of ASD and facilitate the development of precision medicine for ASD.

\section{Acknowledgements}

We are grateful to all of the families at the participating SSC sites, as well as the staff at the Simons Foundation Autism Research Initiative (SFARI). The present study was supported by the Ministry of Education, Culture, Sports, Science and Technology (MEXT) KAKENHI grant numbers 19390171, 16H05242 and 19H03894. MEXT had no role in the design or execution of the study.

\section{Author details}

${ }^{1}$ Tohoku Medical Megabank Organization, Tohoku University, Sendai, Japan. ${ }^{2}$ Graduate School of Medicine, Tohoku University, Sendai, Japan. ${ }^{3}$ RIKEN Center for Advanced Intelligence Project, Tokyo, Japan. ${ }^{4}$ Tohoku University Hospital, Tohoku University, Sendai, Japan. ${ }^{5}$ School of Medicine, Teikyo University, Tokyo, Japan. ${ }^{6}$ School of Medicine, Tohoku Medical and Pharmaceutical University, Sendai, Japan. International Research Institute of Disaster Science, Tohoku University, Sendai, Miyagi, Japan

\section{Data availability}

All data used in the study are available only to those granted access by the Simons Foundation.

\section{Conflict of interest}

The authors declare that they have no conflict of interest.

\section{Publisher's note}

Springer Nature remains neutral with regard to jurisdictional claims in published maps and institutional affiliations.

Supplementary Information accompanies this paper at (https://doi.org/ 10.1038/s41398-020-00951-x).

Received: 14 April 2020 Revised: 15 July 2020 Accepted: 22 July 2020 Published online: 17 August 2020

\section{References}

1. American Psychological Association (2013): Diagnostic and Statistical Manual of Mental Disorders (DSM-5). Washington: American Psychological Association.

2. Geschwind, D. H. \& State, M. W. Gene hunting in autism spectrum disorder: on the path to precision medicine. Lancet Neurol. 14, 1109-1120 (2015).

3. Bailey, A. et al. Autism as a strongly genetic disorder: evidence from a British twin study. Psychol. Med. 25, 63-77 (1995).

4. Lauritsen, M. B., Pedersen, C. B. \& Mortensen, P. B. Effects of familial risk factors and place of birth on the risk of autism: a nationwide register-based study. J. Child Psychol. Psychiatry 46, 963-971 (2005).

5. Gene, S. Gene scoring. 2008. https:/gene.sfari.org/database/gene-scoring/.

6. Eissa, N. et al. Current enlightenment about etiology and pharmacological treatment of autism spectrum disorder. Front. Neurosci. 12, 304 (2018).

7. Traylor, M., Markus, H. \& Lewis, C. M. Homogeneous case subgroups increase power in genetic association studies. Eur. J. Hum. Genet. 23, 863-869 (2015).

8. Mukherjee, S. et al. Genetic data and cognitively defined late-onset Alzheimer's disease subgroups. Mol. Psychiatry. 2018. https://doi.org/10.1038/s41380018-0298-8.

9. Nagel, M., Watanabe, K, Stringer, S., Posthuma, D. \& van der Sluis, S. Item-level analyses reveal genetic heterogeneity in neuroticism. Nat. Commun. 9, 905 (2018).

10. Lavoie-Charland, E., Berube, J. C., Boulet, L. P. \& Bosse, Y. Asthma susceptibility variants are more strongly associated with clinically similar subgroups. $J$. Asthma 53, 907-913 (2016).
11. Chaste, P. et al. A genome-wide association study of autism using the Simons simplex collection: does reducing phenotypic heterogeneity in autism increase genetic homogeneity? Biol. Psychiatry 77, 775-784 (2015).

12. MacQueen, J. Some methods for classification and analysis of multivariate observations. In: Fifth Berkeley Symposium on Mathematical Statistics and Probability. Berkeley: University of California Press, 1967, pp 281-297.

13. World Medical Association. World medical association Declaration of Helsinki: ethical principles for medical research involving human subjects. JAMA 310, 2191-2194 (2013).

14. Fischbach, G. D. \& Lord, C. The simons simplex collection: a resource for identification of autism genetic risk factors. Neuron 68, 192-195 (2010).

15. Beggiato, A. et al. Gender differences in autism spectrum disorders: divergence among specific core symptoms. Autism Res. 10, 680-689 (2017).

16. Kuriyama, $\mathrm{S}$. et al. Pyridoxine treatment in a subgroup of children with pervasive developmental disorders. Dev. Med. Child Neurol. 44, 284-286 (2002).

17. Obara, T. et al. Potential identification of vitamin B6 responsiveness in autism spectrum disorder utilizing phenotype variables and machine learning methods. Sci. Rep. 8, 14840 (2018).

18. Patterson, N., Price, A. L. \& Reich, D. Population structure and eigenanalysis. PLoS Genet. 2, e190 (2006).

19. Nam, J. M. Simple approximation for calculating sample sizes for detecting linear trend in proportions. Biometrics 43, 701-705 (1987).

20. Cutting, D. R., Karger, D. R., Pedersen J. O. \& Tukey J. W. Scatter/gather: a cluster-based approach to browsing large document collections. In: Proceedings of the 15th Annual ACM SIGIR Conference on Research and Development in Information Retrieval. 318-329 (New York: Association for Computing Machinery (ACM), 1992).

21. Raykov, Y. P., Boukouvalas, A., Baig, F. \& Little, M. A. What to do when K-means clustering fails: a simple yet principled alternative algorithm. PLOS ONE 11 e0162259 (2011).

22. Guo, G., Chen, L., Ye, Y. \& Jiang, Q. Cluster validation method for determining the number of clusters in categorical sequences. IEEE Trans. Neural Netw. Learn Syst. 28, 2936-2948 (2017).

23. Sanders, S. J. et al. Multiple recurrent de novo CNVs, including duplications of the 7q11.23 Williams syndrome region, are strongly associated with autism. Neuron 70, 863-885 (2011).

24. Spielman, R. S. \& Ewens, W. J. A sibship test for linkage in the presence of association: the sib transmission/disequilibrium test. Am. J. Hum. Genet $\mathbf{6 2}$, 450-458 (1998).

25. Freidlin, B., Zheng, G., Li, Z. \& Gastwirth, J. L. Trend tests for case-control studies of genetic markers: power, sample size and robustness. Hum. Hered. $\mathbf{5 3}$ 146-152 (2002)

26. Purcell, $\mathrm{S}$. et al. PLINK: a tool set for whole-genome association and population-based linkage analyses. Am. J. Hum. Genet. 81, 559-575 (2002).

27. Wang, K, Li, M. \& Hakonarson, H. ANNOVAR: functional annotation of genetic variants from high-throughput sequencing data. Nucleic Acids Res. 38, e164 (2010).

28. Wang, Y. et al. Genome-wide association study of piglet uniformity and farrowing interval. Front. Genet. 8, 194 (2017).

29. Anttila, $\mathrm{V}$. et al. Analysis of shared heritability in common disorders of the brain. Science 360, eaap8757 (2018).

30. Redies, C., Hertel, N. \& Hubner, C. A. Cadherins and neuropsychiatric disorders, Brain Res. 1470, 130-144 (2012).

31. Varghese, M. et al. Autism spectrum disorder: neuropathology and animal models. Acta Neuropathol. 134, 537-566 (2017).

32. Atsem, S. et al. Paternal age effects on sperm FOXK1 and KCNA7 methylation and transmission into the next generation. Hum. Mol. Genet. 25, 4996-5005 (2016).

33. Barnby, G. et al. Candidate-gene screening and association analysis at the autism-susceptibility locus on chromosome 16p: evidence of association at GRIN2A and ABAT. Am. J. Hum. Genet. 76, 950-966 (2005).

34. Minhas, H. M. et al. An unbalanced translocation involving loss of 10q26.2 and gain of 11q25 in a pedigree with autism spectrum disorder and cerebellar juvenile pilocytic astrocytoma. Am. J. Med. Genet. A 161a, 787-791 (2013).

35. Abou-Donia, M. B., Suliman, H. B., Siniscalco, D., Antonucci, N. \& ElKafrawy, P. De novo blood biomarkers in autism: autoantibodies against neuronal and glial proteins. Behav. Sci. (Basel) 9, E47 (2019).

36. Lo Vasco, V. R. Role of phosphoinositide-specific phospholipase C $\mathrm{n} 2$ in isolated and syndromic mental retardation. Eur. Neurol. 65, 264-269 (2011). 
37. Potkin, S. G. et al. Gene discovery through imaging genetics: identification of two novel genes associated with schizophrenia. Mol. Psychiatry 14, 416-428 (2009).

38. Konopaske, G. T. et al. Dysbindin-1 contributes to prefrontal cortical dendritic arbor pathology in schizophrenia. Schizophr. Res. 201, 270-277 (2018).

39. Openshaw, R. L. et al. JNK signalling mediates aspects of maternal immune activation: importance of maternal genotype in relation to schizophrenia risk. J. Neuroinflamm. 16, 18 (2019).

40. Ikeda, M. et al. Identification of novel candidate genes for treatment response to risperidone and susceptibility for schizophrenia: integrated analysis among pharmacogenomics, mouse expression, and genetic case-control association approaches. Biol. Psychiatry 67, 263-269 (2010)

41. Teng, X. et al. KCTD: a new gene family involved in neurodevelopmental and neuropsychiatric disorders. CNS Neurosci. Ther. 25, 887-902 (2019).

42. Lin, C. H., Huang, M. W., Lin, C. H., Huang, C. H. \& Lane, H. Y. Altered mRNA expressions for $\mathrm{N}$-methyl-D-aspartate receptor-related genes in WBC of patients with major depressive disorder. J. Affect. Disord. 245, 1119-1125 (2019).

43. Kunkle, B. W. et al. Genetic meta-analysis of diagnosed Alzheimer's disease identifies new risk loci and implicates Abeta, tau, immunity and lipid processing. Nat. Genet. 51, 414-430 (2019).
44. Cimino, P. J., Sokal, I., Leverenz, J., Fukui, Y. \& Montine, T. J. DOCK2 is a microglial specific regulator of central nervous system innate immunity found in normal and Alzheimer's disease brain. Am. J. Pathol. 175, 1622-1630 (2009)

45. Sepulveda-Diaz, J. E. et al. HS3ST2 expression is critical for the abnormal phosphorylation of tau in Alzheimer's disease-related tau pathology. Brain 138, 1339-1354 (2015).

46. Ghosh, D., Levault, K. R. \& Brewer, G. J. Relative importance of redox buffers GSH and $\mathrm{NAD}(\mathrm{P}) \mathrm{H}$ in age-related neurodegeneration and Alzheimer diseaselike mouse neurons. Aging Cell 13, 631-640 (2014).

47. Zong, Y. et al. miR-29c regulates NAV3 protein expression in a transgenic mouse model of Alzheimer's disease. Brain Res. 1624, 95-102 (2015).

48. Irimia, $\mathrm{M}$. et al. A highly conserved program of neuronal microexons is misregulated in autistic brains. Cell 159, 1511-1523 (2014).

49. Quesnel-Vallieres, M. et al. Misregulation of an activity-dependent splicing network as a common mechanism underlying autism spectrum disorders. Mol. Cell 64, 1023-1034 (2016).

50. DeMichele-Sweet, M. A. A. et al. Genetic risk for schizophrenia and psychosis in Alzheimer disease. Mol. Psychiatry 23, 963-972 (2018). 